\title{
A boy with refractory cyanosis: pediatric pulmonary arteriovenous malformation case report and literature review
}

\section{Chuqiao Sheng}

Jilin University First Hospital

\section{Chunfeng Yang}

Jilin University First Hospital

\section{Yu Ao}

Jilin University First Hospital

Yumei LI ( $\square$ liyumei201912@126.com )

Jilin University First Hospital https://orcid.org/0000-0002-7705-4950

\section{Case Report}

Keywords: Pulmonary arteriovenous malformation, refractory cyanosis, pharmacotherapy, children, hereditary hemorrhagic telangiectasia, case report

Posted Date: August 4th, 2020

DOl: https://doi.org/10.21203/rs.3.rs-51829/v1

License: (9) This work is licensed under a Creative Commons Attribution 4.0 International License. Read Full License 


\section{Abstract}

Background: Pulmonary arteriovenous malformation is rare and is defined as the presence of an abnormal connection between the pulmonary artery and vein. Pulmonary arteriovenous malformation can manifest with no obvious clinical symptoms and is only found incidentally on pulmonary imaging examinations. The clinical signs vary according to the shunt flow in proportion to the malformation area. In severe cases, symptoms such as exertional dyspnea, cyanosis, hemoptysis or even sudden death can occur. Untreated pulmonary arteriovenous malformation may result in cardiac failure or rupture of the aneurysmal fistula.

Case presentation: We report the case of a 1-year-old boy who presented with refractory cyanosis and a transcutaneous oxygen saturation level of 70-76\%.Pulmonary arteriovenous malformation was detected on computed tomography angiogram. We chose surgical resection of the diseased lung as the treatment. The boy had a good prognosis and was followed up for 3 months without recurrence. The analysis of this case and a review of the literature improved our understanding of pulmonary arteriovenous malformation.

Conclusion: Pulmonary arteriovenous malformation should be considered when unexplained conditions such as hypoxia and hemoptysis are encountered or when pulmonary arteriovenous malformation-like mass changes are visible on chest imaging. The early identification of the problem, diagnosis, treatment and follow-up are helpful for reducing trauma and for improving the long-term outcome of children with pulmonary arteriovenous malformation.

\section{Background}

Pulmonary arteriovenous malformation (PAVM), a rare pulmonary vascular lesion, was first discovered by Churton during an autopsy of a 12-year-old boy in 1897[1]. The incidence of PAVM is 2-3 per 100000 population. The male to female ratio varied from 1:1.5 to 1.8 in several series[2]. However, recent reports from Japan[3] indicate a higher frequency of approximately 38 per 100 000. PAVM may manifest with no obvious clinical symptoms and is only found incidentally on pulmonary imaging examinations $[4,5]$. In severe cases, symptoms such as exertional dyspnea, cyanosis, hemoptysis or even sudden death can occur [6].

\section{Case Presentation}

A 1-year-old boy was hospitalized due to a cough for 15 days and cyanotic lips and nails for 5 days. Prior to hospital admission, he was diagnosed with severe pneumonia in a local hospital and given oxygen inhalation therapy. However, the child's cyanosis was not relieved, and his transcutaneous oxygen saturation fluctuated between 70 and $76 \%$. Therefore, he was transferred to our center. During the course of the disease, the child slept well and consumed a normal diet. He was born to a G2P2 mother by a fullterm cesarean delivery and weighed $3.4 \mathrm{~kg}$ at birth. His height and weight percentiles, vital signs, and 
results of other examinations of body systems were within normal limits. The family denied epistaxis, mucocutaneous telangiectasias, or any first-degree relative with hereditary hemorrhagic telangiectasia, and they denied the intrusion of foreign objects or any history of repeated respiratory infections or congenital heart disease. Normally, the child had good tolerance to activity. He lived in an urban area, and any history of poison or well water intake was eliminated. The physical examination revealed the following: poor general condition, lip cyanosis, and mild acropachy. No abnormal findings were detected with regard to the respiratory sounds and cardiovascular system examination. His hemoglobin level was $124 \mathrm{~g} / \mathrm{L}$, and his hematocrit level was $51 \%$. The arterial blood gas analysis showed the following results: $\mathrm{pH} 7.391, \mathrm{PCO}_{2} 27.1 \mathrm{mmHg}, \mathrm{PO}_{2} 30 \mathrm{mmHg}$, and lactate level $1.49 \mathrm{mmol} / \mathrm{L}$. His electrocardiographic and echocardiographic examination findings and other routine laboratory analyses yielded normal results. On chest $\mathrm{CT}$, a hyperdense area with irregular contours in the medial basal region of the left lung was observed. Signs of PAVM were confirmed on ensuing chest computed tomography angiography (CTA) (fig. 1, arrows).

His transcutaneous oxygen saturation level measured on room air was $75 \%$, and when $100 \%$ oxygen was delivered, his blood oxygen saturation level did not increase. This refractory hypoxia and the pulmonary imaging findings led us to strongly suspect PAVM. In view of the severity of the hypoxia, the patient needed immediate intervention. However, embolotherapy was impossible in this patient because he was young and had limited femoral arteriovenous access. After consulting with his family members, thoracoscopic surgery was performed to remove the diseased lung. Intraoperatively, angioma-like lesions that covered a large range with unclear boundaries were observed in the inferior lobe of the left lung (fig. 2). The left inferior lung ligament had many small blood vessels. The clinical diagnosis was intraoperatively confirmed as arteriovenous malformation of the left inferior pulmonary lobe, and the left inferior lobe was resected. The pathological report confirmed PAVM (fig. 3).

Ventilator-assisted ventilation was withdrawn $20 \mathrm{~h}$ postoperatively, and his transcutaneous oxygen saturation level increased to $92-95 \%$ in the patient without inhaled oxygen. A postoperative pulmonary CT on day 3 revealed inflammatory absorption and small pleural effusion. Moreover, no abnormalities were found on cardiac ultrasound, head magnetic resonance angiography (MRA) or magnetic resonance venography (MRV). After 12 days of hospitalization, the child showed no cough or dyspnea and had a transcutaneous oxygen saturation of $95-97 \%$ on room air; then, he was discharged. Noncontrast CT at the 3-month follow-up showed no residual PAVM sac. The prognosis suggests that our treatment was timely and accurate. This study was approved by the Ethics Committee of the First Hospital of Jilin University. \{Approval No. 2019-252\}.

\section{AETIOLOGY}

PAVM refers to the dilation and tortuosity of the pulmonary artery, its branches and corresponding pulmonary veins or the formation of abnormal communicating branches in a cavernous hemangioma[1]. Depending on the affected communicating branch, pulmonary vascular malformations are often classified as pulmonary artery malformations, pulmonary venous malformations and PAVMs, of which 
PAVMs are the most common type. In the existing literature, PAVM is also known as pulmonary arteriovenous fistula, hemorrhagic telangiectasia with pulmonary artery aneurysm, pulmonary vasodilation, etc.[7,8]. Over $80 \%$ of PAVMs are caused by congenital dysplasia. The congenital factors are considered to be abnormal TGF- $\beta$ signaling during the development of the embryonic pulmonary vasculature, a septal formation disorder between the pulmonary artery branches and pulmonary venous plexuses, defects in capillary development, and the direct return of venous blood in the pulmonary artery to the left heart via the pulmonary vein without capillary oxygenation, which is hemodynamically classified as an "extracardiac" right-to-left shunt[9]. According to some scholars, PAVM in the elderly population is often secondary to hereditary hemorrhagic telangiectasia (HHT; also known as Osler-WeberRendu syndrome) $[4,10]$. Secondary or acquired PAVM, although very rare, has been reported in the literature. Causes of secondary PAVM include postoperative complications of congenital heart disease in children (including the classic Glenn shunt [11]), chest trauma, long-standing hepatic cirrhosis, mitral stenosis, and infections (actinomycosis and schistosomiasis) [12]. Since our patient had no relevant family history, epistaxis, telangiectasia or visceral organ involvement, HHT disease was not considered. Therefore, the diagnosis of the patient was presumed to be congenital PAVM.

\section{PATHOLOGY and PATHOPHYSIOLOGY}

Most solitary PAVMs are seen in the bilateral lower lobes, with the left lower lobe being the most common location[13]. Most of the lesions are either subpleural or partially embedded in the outer third of the lung parenchyma. Approximately $50-75 \%$ of PAVMs are single lesions, and the incidence of bilateral PAVMs ranges from $8 \%-20 \%[14]$. Depending on the pathological features, PAVMs can be classified into three types[15]: (1) the simple type accounts for approximately $80-90 \%$ of PAVMs and refers to the communication of a single feeding pulmonary artery with a single draining pulmonary vein, without dilated cyst septation. This type of lesion can appear as either a solitary lesion or multiple lesions; (2) the complex type accounts for approximately $20 \%$ of all PAVMs and refers to the communication of two or more feeding pulmonary arteries with draining pulmonary veins, often with cyst septation or tortuous dilated vessels; and (3) the diffuse type, which is rare and refers to the interconnection and diffuse distribution of numerous tiny pulmonary arteries and veins (tiny fistulas), without cyst formation. In the patient in the present case, under thoracoscopy, a large range of angioma-like lesions were observed intraoperatively, with the presence of tortuous dilated vessels, which is consistent with the pathological manifestations of a complex PAVM.

\section{CLINICAL FINDINGS}

Among the adult population, the typical clinical manifestations of PAVM include exertional dyspnea, cyanosis, hemoptysis, and clubbing of the fingers/toes[15,16]. In the event of pleural involvement, chest pain and cough may occur. Among the pediatric population, the clinical manifestations are not typical, and patients may primarily present with cyanosis and hemoptysis. Clinical signs vary according to the amount of shunt in proportion to the number and size of the fistulae: at low shunt flows, pediatric patients may show no obvious symptoms and will probably exhibit the onset of symptoms during 
adulthood. At high shunt flows, more than $20 \%$ of patients may show a series of hypoxemic symptoms and even develop heart failure. Approximately $10 \%$ of patients may have life-threatening massive hemoptysis[17]. An increased growth rate of a PAVM has been attributed to increased blood volume and cardiac output, which leads to increased pulmonary blood flow, preferentially across the low-resistance PAVM. In addition, ectopic thromboses such as stroke, cerebral embolism and abscess may occur because the venous blood enters the systemic circulation directly without passing through the alveolar capillaries[18,19].

\section{DIAGNOSIS}

In a patient with PAVM, we can observe probable marked elevations of erythrocytes and hemoglobin caused by prolonged chronic hypoxia, while children with repeated hemoptysis often present with anemia. The blood gas analysis revealed refractory hypoxemia without carbon dioxide retention and normal lactic acid levels. Regarding imaging examinations[20], pulmonary CT is more sensitive than conventional chest X-ray for PAVM detection and can be used to identify almost all PAVMs. Thus, pulmonary CT examinations are the preferred diagnostic imaging method. On CT, we can observe circular or oval nodules with uniform density, smooth and clear boundaries, and occasional lobulation; however, these nodules also need to be differentiated from early tumors, lung abscesses, miliary tuberculosis, bronchial foreign bodies, etc. CTA can stereoscopically display 3D images of the PAVM to show highdensity intrapulmonary nodular or mass shadows. Simultaneous enhancement of the lesions on adjacent large vessels is possible and is characterized by a "vascular pedicle" sign or "aneurysm" sign and premature imaging of the left atrium[20]. Moreover, echocardiography is highly necessary since congenital pulmonary vascular malformations are often accompanied by cardiac anomalies.

Additionally, head MRI or MRA+MRV can rule out the presence or absence of intracranial embolisms. The patient in the present case showed refractory cyanosis as the initial symptom and was diagnosed with pneumonia by a local hospital. However, we could see very limited inflammation and seemingly quasicircular areas on pulmonary CT. Further CTA examinations demonstrated the entry of the contrast agent into a lesion-like mass from the artery, so the possibility of a local vascular mass was high. Additionally, the patient had severe hypoxia and rather large lesions, so the presence or absence of accompanying cardiovascular malformations and cerebral embolism was suspected. Hence, we performed head MRI and echocardiography and fortunately found no abnormalities.

Adolescents with repeated occurrences of hemoptysis, dyspnea, cyanosis and clubbing of the fingers/toes can be diagnosed with PAVM based on characteristic pulmonary imaging findings after congenital cardiovascular diseases have been excluded. In infants and young children, ruling out bronchial foreign bodies and congenital pulmonary dysplasia is necessary. Where conditions permit, pulmonary CTA should be performed as soon as possible. PAVM needs to be considered in the differential diagnosis of common pediatric respiratory and circulatory problems and conditions (e.g., pulmonary tuberculosis, lung space-occupying lesions, cyanotic congenital heart diseases). The patient in the present case had no previous history of repeated cyanosis. After respiratory onset, refractory cyanosis occurred following an improvement in his cough. Moreover, his pulmonary imaging findings 
differed distinctly from those of common pneumonia. Therefore, hypoxia caused by other factors needed to be considered. Further CTA examinations suggested PAVM, and the surgical and pathological findings confirmed the imaging-based diagnosis.

\section{TREATMENT}

Therapeutically, the current treatments for PAVM include transcatheter embolization (TCE), surgery and pharmacotherapy. At present, TCE is considered the gold standard because it reduces the risk of paradoxical emboli and other complications[21.22]. TCE has satisfactory efficacy for simple and partial complex PAVMs. According to some studies, the decision to adopt TCE intervention depends on the feeding artery diameter (FAD), and the occlusion method selected also varies somewhat based on the FAD[23-26]. For patients with confirmed PAVM, angiography and TCE are recommended regardless of the presence or absence of pulmonary symptoms when the FAD is $\geq 2 \mathrm{~mm}$. When the FAD is $\leq 2 \mathrm{~mm}, T C E$ is recommended for patients with severe hypoxemia, paradoxical embolisms (e.g., cerebral abscess, stroke) and hemoptysis. For asymptomatic patients with a FAD $\leq 2 \mathrm{~mm}$, annual follow-up contrast-enhanced pulmonary CT reexaminations are recommended. Additionally, pulmonary CT monitoring is recommended every 3-5 years to determine whether the PAVM has increased in size. In the present case, the patient was very young, and he had severe clinical symptoms and limited vascular access. Thus, it was impossible to perform interventional therapy, and surgical resection of the diseased lung was adopted instead. Surgery is a radical measure, with techniques including ligation, segmentectomy/lobectomy, local or total pneumonectomy, etc. For patients in whom embolotherapy repeatedly fails, who have life-threatening hemorrhage resulting from PAVM rupture, or who are ineligible for TCE due to medical limitations, thoracoscopic surgery can be performed to remove the lesion. In the case of diffuse PAVFs, a lung transplant is required. However, surgical therapy is not the optimal choice because it is quite traumatic to children and is detrimental to their thoracic development.

Pharmacotherapy is an adjuvant treatment to TCE and surgery and includes oral estrogens and antifibrinolytic and antiangiogenic agents (octreotide, desmopressin). However, there are no recommendations for the dosages of these drugs in pediatric patients.

\section{OUTCOME}

PAVM is a progressive disease that can be effectively treated by embolotherapy [27]. A common postoperative issue, however, is recanalization of the fistula, especially within the pediatric population. The incidence of fistula recanalization with aging is approximately $5-10 \%[28]$. Given the less than $2 \%$ recurrence rate after surgical resection and the low incidence of intraoperative complications and mortality, active intervention is effective at improving the long-term outcome. However, if the disease remains untreated, it may result in cardiac failure and infective endocarditis, thereby leading to rupture of the aneurysmal fistula.

\section{Discussion And Conclusion}


The incidence of PAVM is low, especially in pediatric patients. The possibility of PAVM should be considered when unexplained conditions such as hypoxia and hemoptysis are encountered or when PAVM-like mass changes are visible on pediatric chest imaging. Early lesion identification, diagnosis, treatment and follow-up are helpful for reducing trauma and improving the long-term outcome of children with PAVM.

There are currently no recommended dosages of therapeutic drugs in pediatric patients; therefore, further research is being developed to determine whether $\beta$-receptor blockers can be used as adjuvant therapy to reduce surgical trauma.

\section{Abbreviations}

\begin{tabular}{ll} 
computed tomography angiogram & CTA \\
\hline feeding artery diameter & $\mathrm{FAD}$ \\
\hline hereditary hemorrhagic telangiectasia & $\mathrm{HHT}$ \\
\hline millimeter(s) of mercury & $\mathrm{mmHg}$ \\
\hline magnetic resonance angiography & $\mathrm{MRA}$ \\
\hline magnetic resonance venography & $\mathrm{MRV}$ \\
\hline pulmonary arteriovenous malformations & $\mathrm{PAVMs}^{-}$ \\
\hline partial pressure of carbon dioxide & $\mathrm{PCO}_{2}$ \\
\hline partial pressure of oxygen & $\mathrm{PO}_{2}$ \\
\hline transcatheter embolization & $\mathrm{TCE}^{2}$
\end{tabular}

\section{Declarations}

\section{Funding:}

No funding was obtained for this study.

\section{Conflict of Interest Statement:}

The authors have indicated they have no conflicts of interest relevant to this article to disclose.

\section{Authors Contributions and Consent for Publication:}

Dr Chuqiao Sheng conceptualized the study, drafted the initial manuscript, and reviewed and revised the manuscript. Dr Chunfeng Yang and Dr Yu Ao collected data, carried out the initial analyses, and reviewed and revised the manuscript. Dr Yumei Li coordinated and supervised data collection, and critically 
reviewed the manuscript. All authors have read and approved the final manuscript as submitted and agree to be accountable for all aspects of the work in ensuring that questions related to the accuracy or integrity of any part of the work are appropriately investing and resolved.

\section{Acknowledgments:}

Written informed consent was obtained from the patient's legal guardian for the publication of this case report and any accompanying images. A copy of the written consent is available.

\section{Availability of data and materials:}

All the data and material are availability. The datasets used and analysed during the current study available from the corresponding author on reasonable request.

\section{References}

[1] Churton T.Multiple aneurysms of the pulmonary artery(J).Br Med.1897;1:1223.

(https://www.researchgate.net/publication/287013238_Multiple_aneurysm_of_pulmonary_artery)

[2] Chiang K,Wang A H,Chang E.Pulmonary Arteriovenous Malformation(J). American journal of respiratory and critical care medicine, 2011, 184(5).DOI: 10.1164/rccm.201101-0024IM

[3] Nakayama M , Nawa T , Chonan T , et al. Prevalence of Pulmonary Arteriovenous Malformations as Estimated by Low-Dose Thoracic CT Screening(J). Internal Medicine, 2012, 51(13):1677-1681.DOI: 10.2169/internalmedicine.51.7305

[4] Angriman F, Ferreyro BL, Wainstein EJ, et al. Pulmonary arteriovenous malformations and embolic complications in patients with hereditary hemorrhagic telangiectasia(J). Arch Bronconeumol. 2014 Jul;50(7):301-4. D0Iه10.1016/j.arbr.2014.05.009

[5] Caleb J.Heiberger,Mark J.brown,Divyajot Sandhu,et al.Pulmonary Arteriovenous Malformations: A Rare Cause of Ischemic Stroke(J).Cureus. 2019,11(7):e5141. DOI: 10.7759/cureus.5141.

[6] Jutant EM, Puyo P, El Hajjam M,et al.Severe,chronic cough caused by pulmonary arteriovenous malformations in a patient with hereditary haemorrhagic telangiectasia:case report(J). BMC Pulm Med,2015(15):28. DOI: 10.1186/s12890-015-0024-0

[7] Rotenberg C,Bonay M,El Haijam,et al.Effect of pulmonary arteriovenous malformations on the mechanical properties of the lungs(J).BMC Pulm Med.2017;17(1):64. DOI:10.1186/s12890-017-0411-9.

[8] Sloan RD, Cooley RN. Congenital pulmonary arteriovenous aneurysm(J). Am J Roentgenol Radium Ther Nucl Med. 1953;70(2):183. DOI: 10.1097/00003072-198102000-00012 
[9] Vorselaars V M M , Velthuis S, Mager $\mathrm{J} J$, et al. Direct haemodynamic effects of pulmonary arteriovenous malformation embolisation(J). Netherlands Heart Journal, 2014, 22(7-8):328-333.DOI: $10.1007 / \mathrm{s} 12471-014-0539-7$

[10] Fuchizaki U, Miyamori H, Kitagawa S, et al. Hereditary haemorrhagic telangiectasia (Rendu-OslerWeber disease) (J). Lancet. 2003;362(9394):1490.DOI: 10.1016/S0140-6736(03)14696-X

[11] Mathur M, Glenn WW. Long-term evaluation of cava-pulmonary artery anastomosis(J). Surgery. 1973;74(6):899. PMID:4127196

[12] Tamura J, Tawarahara K,Suwa K,et al.Case report:a case of pulmoary arteriovenous fistula benefited by transcatheter embolotherapy(J).Nihon Naika Gakkai Zasshi,2011,100(7):1972-

1974. DOI:10.2169/naika.100.1972.

[13] Mager JJ, Overtoom TT, Blauw H, et al. Embolotherapy of pulmonary arteriovenous malformations: long-term results in 112 patients $(\mathrm{J})$. J Vasc Interv Radiol. 2004 May;15(5):451-6.

DOI:10.1097/01.rvi.0000126811.05229.b6

[14] Cartin-Ceba R , Swanson K L , Krowka M J . Pulmonary Arteriovenous Malformations(J). CHEST Journal, 2013, 144(3):1033. DOI:10.1378/chest.12-0924

[15] Wong HH, Chan RP, Klatt R, et al. Idiopathic pulmonary arteriovenous malformations: clinical and imaging characteristics (J). Eur Respir J. 2011 Aug;38(2):368-75.DOI区

10.1378/chest.132.4_MeetingAbstracts.625

[16] Shin JH, Park SJ, Ko GY, et al. Embolotherapy for pulmonary arteriovenous malformations in patients without hereditary hemorrhagic telangiectasia(J). Korean J Radiol. 2010 May-Jun;11(3):312-9. DOI区 10.3348/kjr.2010.11.3.312

[17] Velthuis S, Buscarini E, van Gent MW, et al. Grade of pulmonary right-to-left shunt on contrast echocardiography and cerebral complications: a striking association(J). Chest. 2013 Aug;144(2):5428. DOIه10.1378/chest.12-1599

[18] Shaikh Z , Yaqubi B , Rathi A, et al. Pulmonary Arteriovenous Malformation Causing Brain Abscess in a Patient With Hereditary Hemorrhagic Telangiectasia(J). Chest, 2017, 152(4):A909.DOI:10.1016/j.chest.2017.08.944.

[19] Rohit M,Gupta A. Pulmonary arteriovenous malformation with actinomycotic brain abscess: a rare association(J).Pediatr Neurol,2014,50(6):660-661. DOl囚10.1016/j.pediatrneurol.2014.01.045

[20] Leitman EM,McDermott S.Pulmonary arteries: imaging of pulmonary embolism and beyond(J).Cardiovasc Diagn Ther.2019.9(suppl 1):S37-S58.DOI: 10.21037/cdt.2018.08.05 
[21] Fu Z,Liang Y,Zhao W,et al.Safety and efficacy of transcatheter embolization in patients with massive hemoptysis due to intercostal pulmonary venous shunts(J).Radiol Med.2019,124(7):588-594.DOI: 10.1007/s11547-019-01020-0.

[22] Saboo S S , Chamarthy M , Bhalla S, et al. Pulmonary arteriovenous malformations: diagnosis(J). Cardiovascular diagnosis and therapy, 2018, 8(3):325-337.DOI区 10.21037/cdt.2018.06.01

[23] Trerotola SO, Pyeritz RE. PAVM embolization: an update(J). AJR Am J Roentgenol. 2010 Oct;195(4):837-45.DOI:10.2214/AJR.10.5230.

[24] Remy-Jardin M , Dumont $P$, Brillet $P Y$, et al. Pulmonary Arteriovenous Malformations Treated with Embolotherapy: Helical CT Evaluation of Long-term Effectiveness after 2-21-Year Follow-up1(J). Radiology, 2006, 239(2):576-585. DOIه 10.1148/radiol.2391050333

[25] Hsu C T , Kwan G N C , Thompson S A, et al. Embolisation for pulmonary arteriovenous malformation(J). Cochrane Database of Systematic Reviews, 2012, 8(8):CD008017.DOI:10.1002/14651858.CD008017.pub3.

[26] Andersen P E , Duvnjak S , Gerke O , et al. Long-Term Single-Center Retrospective Follow-Up After Embolization of Pulmonary Arteriovenous Malformations Treated Over a 20-year Period: Frequency of Recanalization with Various Embolization Materials and Clinical Outcome $(\mathrm{J})$. CardioVascular and Interventional Radiology, 2019.DOI:10.1007/s00270-019-02204-x.

[27] Andersen PE.Tørring PM. Duvnjak S.et al.Pulmonary arteriovenous malformations: a radiological and clinical investigation of 136 patients with long-term follow-up(J).Clin Radiol,2018,72(11):951-957. DOI: 10.1016/j.crad.2018.07.096.

[28] Andersen PE , Kjeldsen A D . Long-Term Follow-up After Embolization of Pulmonary Arteriovenous Malformations with Detachable Silicone Balloons(J). Cardiovasc Intervent Radiol, 2008, 31 (3):569574.DOIه10.1007/s00270-007-9256-6

\section{Figures}




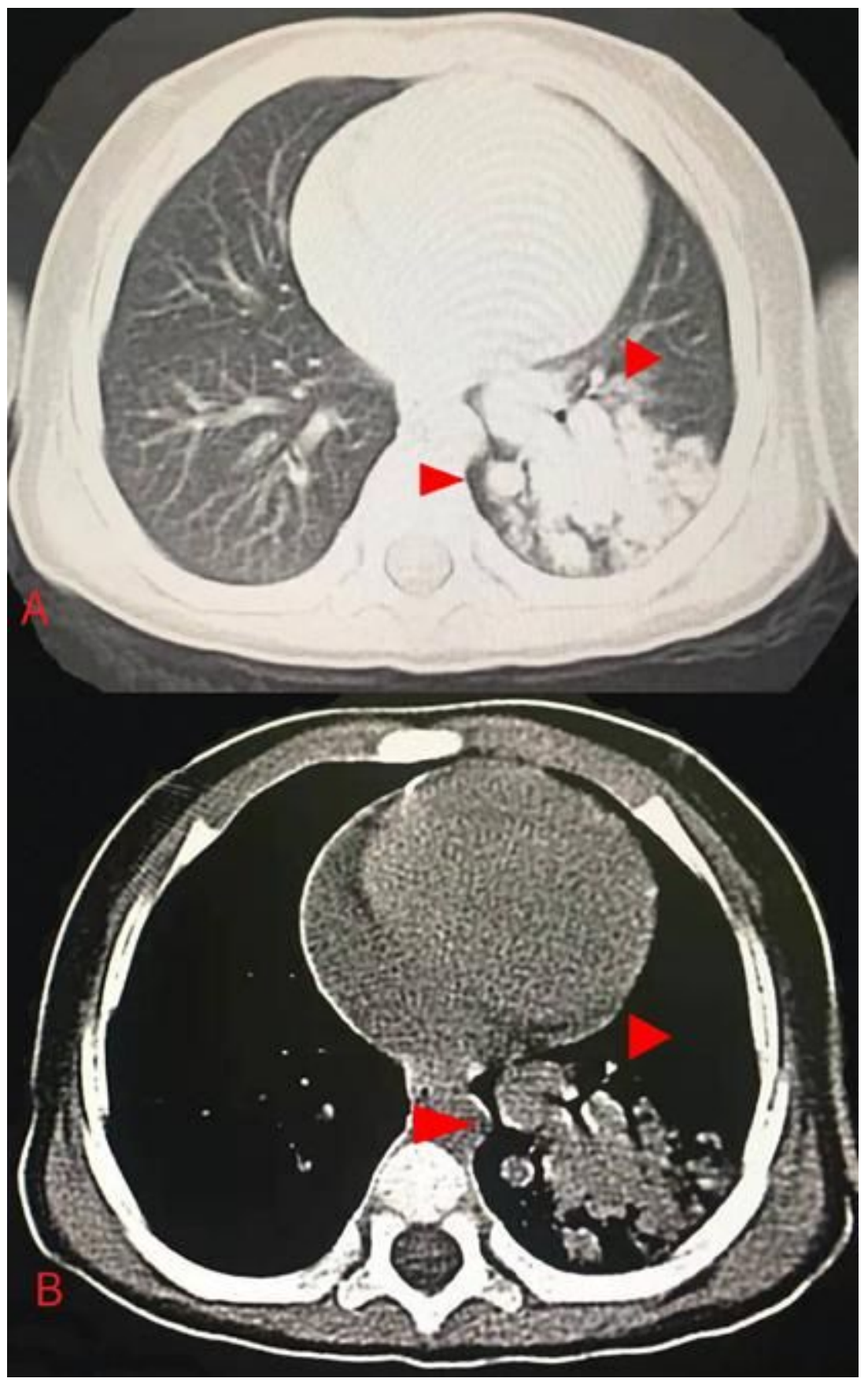

Figure 1

(A, B) The pulmonary computed tomography angiogram (CTA) revealed a probable arteriovenous malformation of the inferior lobe of the left lung. 


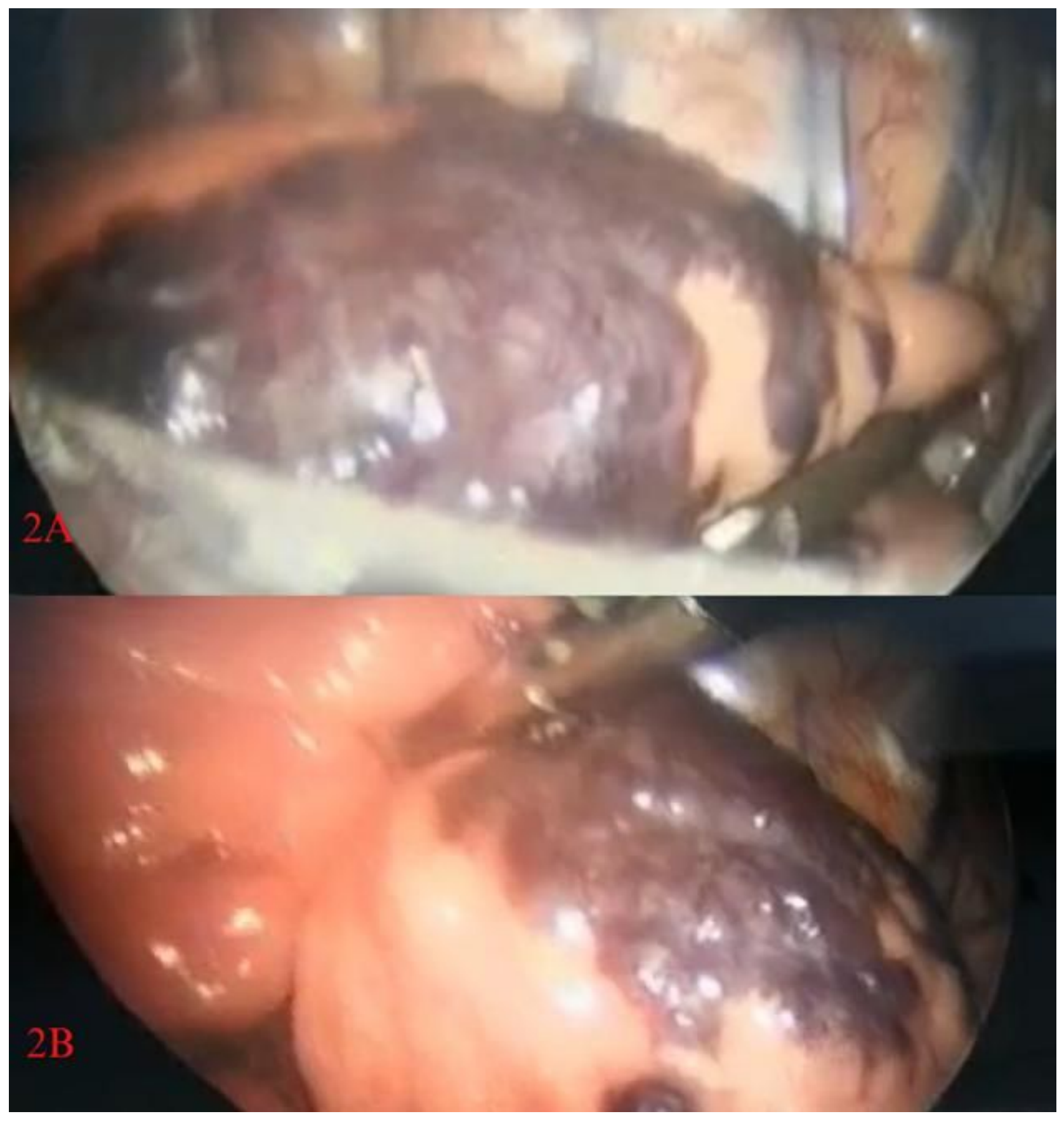

\section{Figure 2}

(A, B) Large-area angioma-like lesions covering the surface of normal lung tissues (lower inferior lung), as seen with thoracoscopy. 


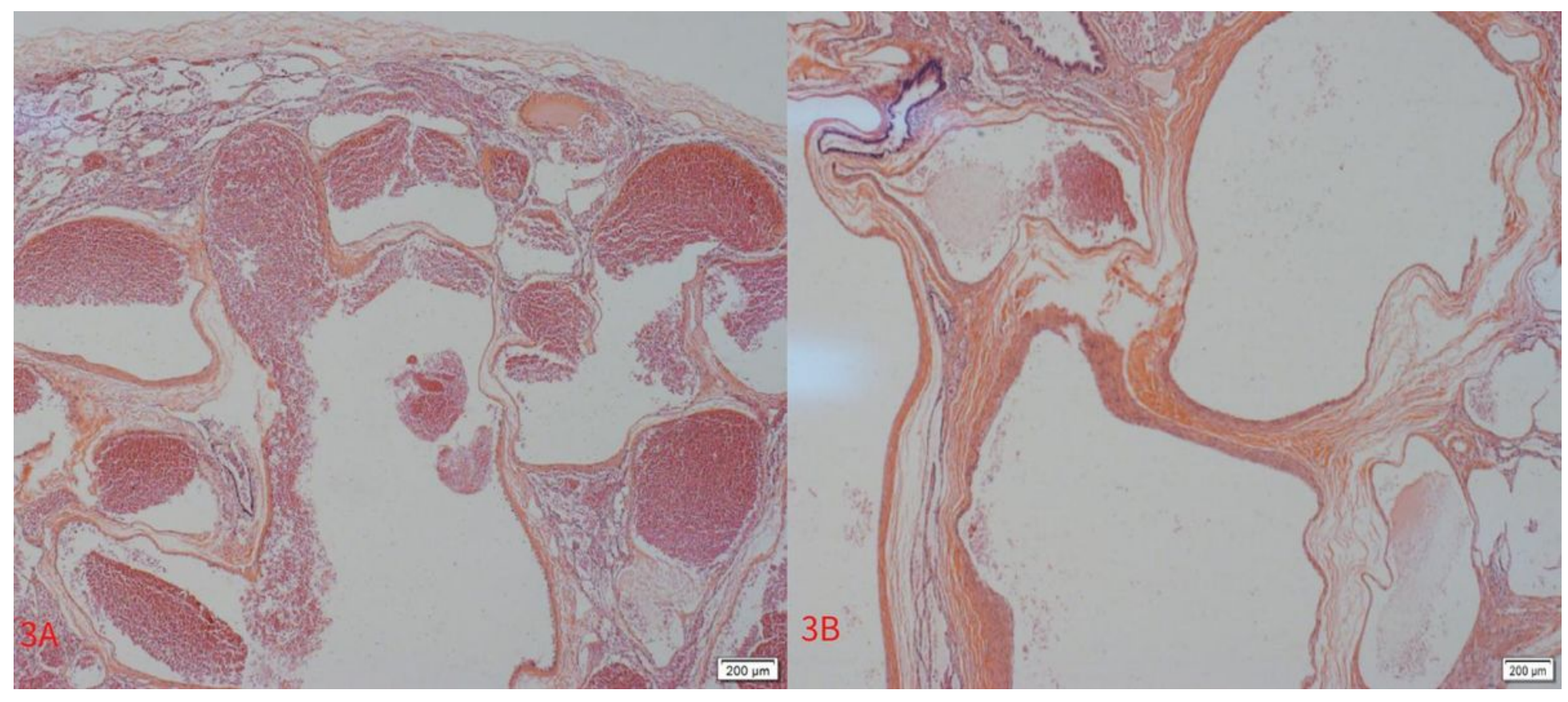

\section{Figure 3}

(HE staining, 10x) Irregularly dilated blood vessels, some of which manifest angioma-like hyperplasia, with most of the surrounding alveolar spaces being congested. Conforms to vascular malformation.

\section{Supplementary Files}

This is a list of supplementary files associated with this preprint. Click to download.

- CAREchecklist.pdf 